\title{
A Case Report of Polycystic Kidney Disease
}

\author{
Henry Mayala
}

\begin{abstract}
Background: Polycystic kidney disease is a disorder in which clusters of cysts develop primarily within your kidneys. Cysts are noncancerous round sacs containing water like fluid. Polycystic kidney disease isn't limited to your kidneys, although the kidneys are the most severely affected organs. The disease can cause cysts to develop in your liver and elsewhere in your body. A common complication of polycystic kidney disease is high blood pressure.

Case presentation: In this report a 49 years old man who is a known diabetic type 2 and hypertensive for 3 years was treated as an outpatient for the first time at Sanitas Hospital with the presenting complains of right flank pain and generalized headache, with the family history of diabetes mellitus type 2. Clinical and imaging findings are suggestive of polycystic kidney disease

Conclusion: Polycystic kidney disease is an important cause of secondary hypertension in children and in adults, it is responsible for cardiovascular and kidney complications, thus making it necessary for this case report.
\end{abstract}

Key words: polycystic kidney disease, abdominal pelvic ultrasound, abdominal CT-scan

\section{INTRODUCTION}

Polycystic kidney disease is a disorder in which clusters of cysts develop primarily within your kidneys. Cysts are noncancerous round sacs containing water like fluid. Polycystic kidney disease isn't limited to your kidneys, although the kidneys are the most severely affected organs. The disease can cause cysts to develop in your liver and elsewhere in your body. A common complication of polycystic kidney disease is high blood pressure. ${ }^{1}$

One third of people older than 50 years develop renal cysts. Although most are simple cysts, renal cystic disease has multiple etiologies. The broad categories of cystic disease include the following:

- Developmental- multicystic dysplastic kidney (MCDK)

- Genetic-Autosomal recessive polycystic kidney disease (ARPKD),autosomal dominant polycystic kidney disease(ADPKD), juvenile nephronopthisis (JNPHP), medullary cystic kidney disease(MCKD), glomerulocystic kidney disease.

- Cysts associated with systemic disease- Von hippel-Lindau syndrome, tuberous sclerosis

- Acquired- simple cysts, acquired cystic renal disease, medullary sponge kidney(MSK)

- Malignancy-cystic renal cell carcinoma (RCC)

The most common larger cysts include acquired cysts, simple cyst and cyst associated with ADPKD. Smaller cysts characterize ARPKD, JNPHP, MCKD and MSK. In adults, renal angiomyolipomas and RCC may have cystic components. ${ }^{2}$

Polycystic kidney disease- presents with ${ }^{3}$

- High blood pressure

- Back pain or flank pain.

- An increase in size of the abdomen

- Blood in urine

- Frequent bladder or kidney infection

- Or may be asymptomatic. 


\section{CASE REPORT}

We present a case report of a 49 years old man who is a known diabetic type 2 and hypertensive for 3 years was treated as an outpatient for the first time at Sanitas Hospital with the presenting complains of right flank pain, generalized headache, and an increase in size of the abdomen.He has no history of admission, nor drug or food allergy. Moreover there is a family history of diabetes mellitus type 2 , his mother is diabetic. The patient's blood pressure was $180 / 100 \mathrm{mmHg}$. The patient underwent the following investigations: EKG, Echocardiogram, RBG,HbA1c, abdominal pelvic USS, and abdominal CT-scan, lipid profile, liver function test and renal function test.

Abdominal pelvic ultrasound revealed multiple simple cysts on the right and left kidneys respectively, and also fatty liver as shown in figures 1,2 and 3.

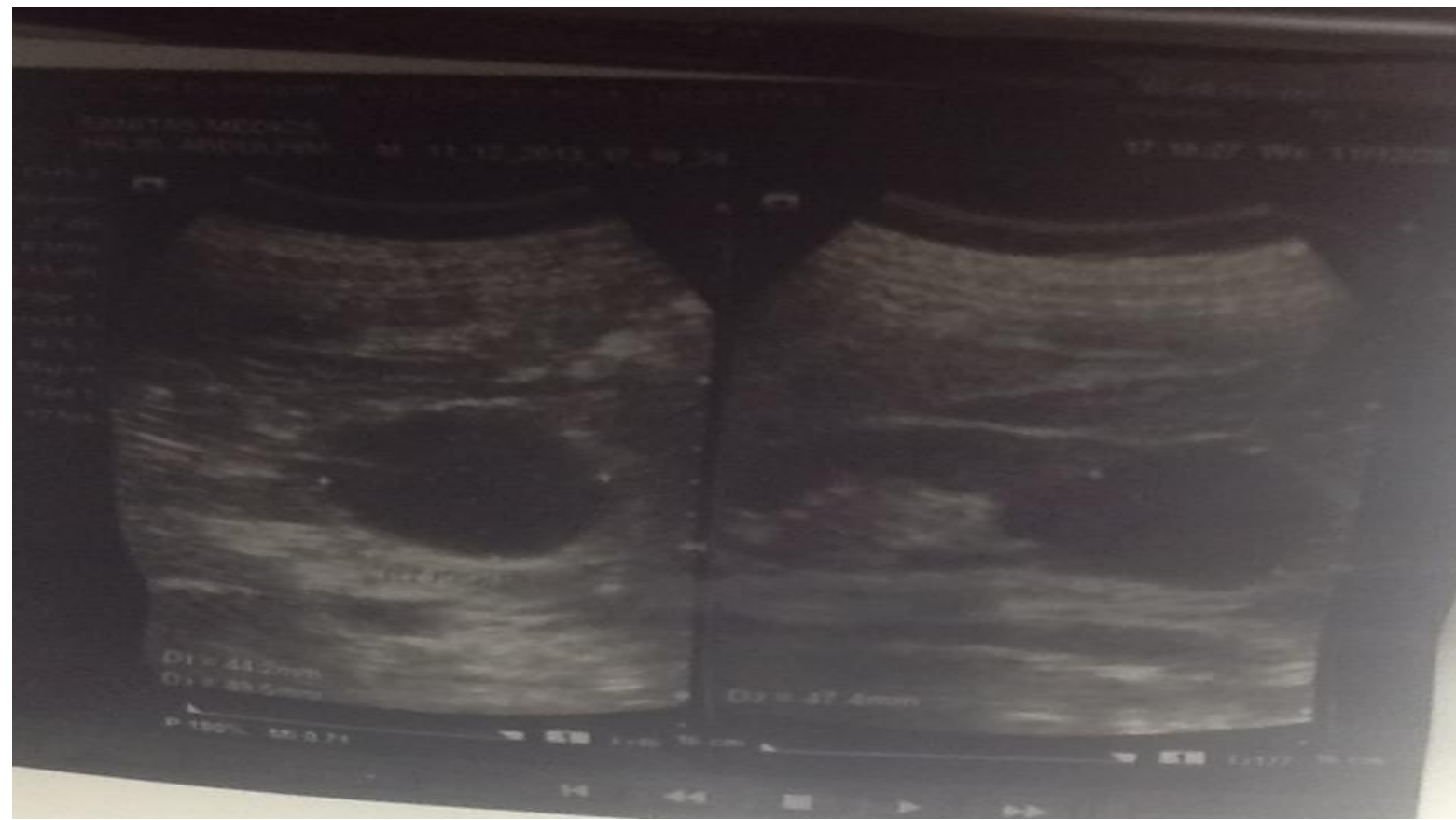

Figure 1: showing cysts on the left and right kidneys respectively through abdominal pelvic ultrasound

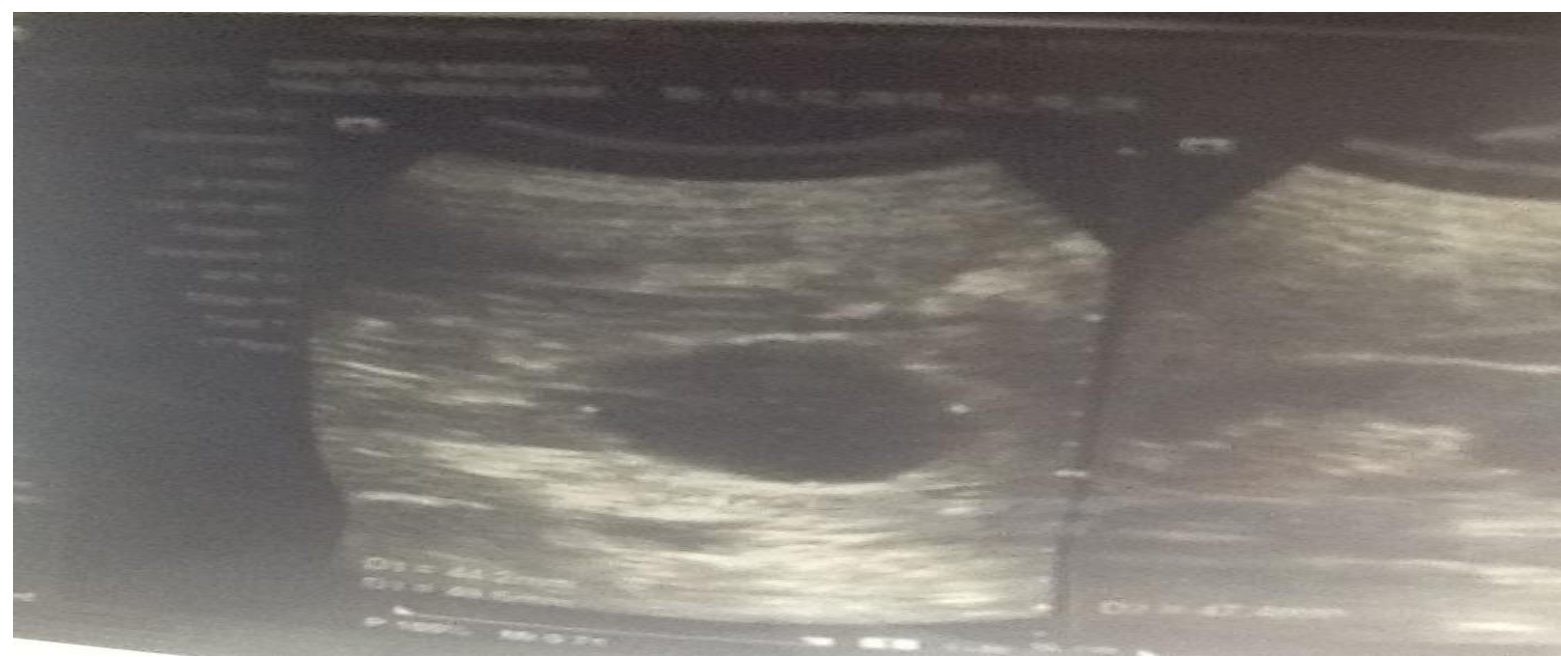

Fig 2: showing a cyst on the right kidney through abdominal pelvic ultrasound

Abdominal Ct-scan also revealed multiple bilateral simple renal cysts, right renal calcified stone $(0.64 * 0.35 \mathrm{~cm})$ and diffuse hepatic fatty infiltration. Fig.3 


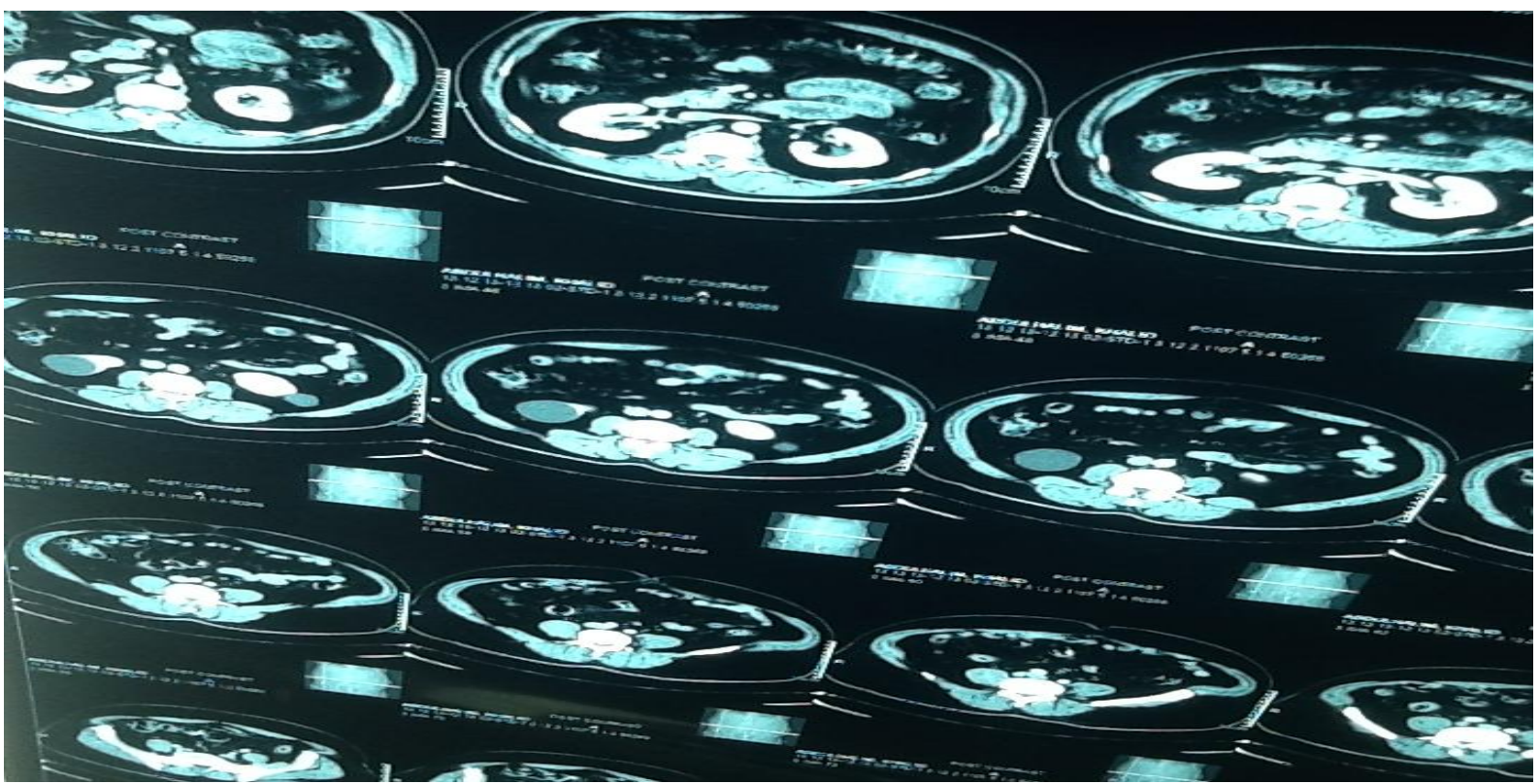

Figure 3: CT-scan showing multiple cysts on the kidneys

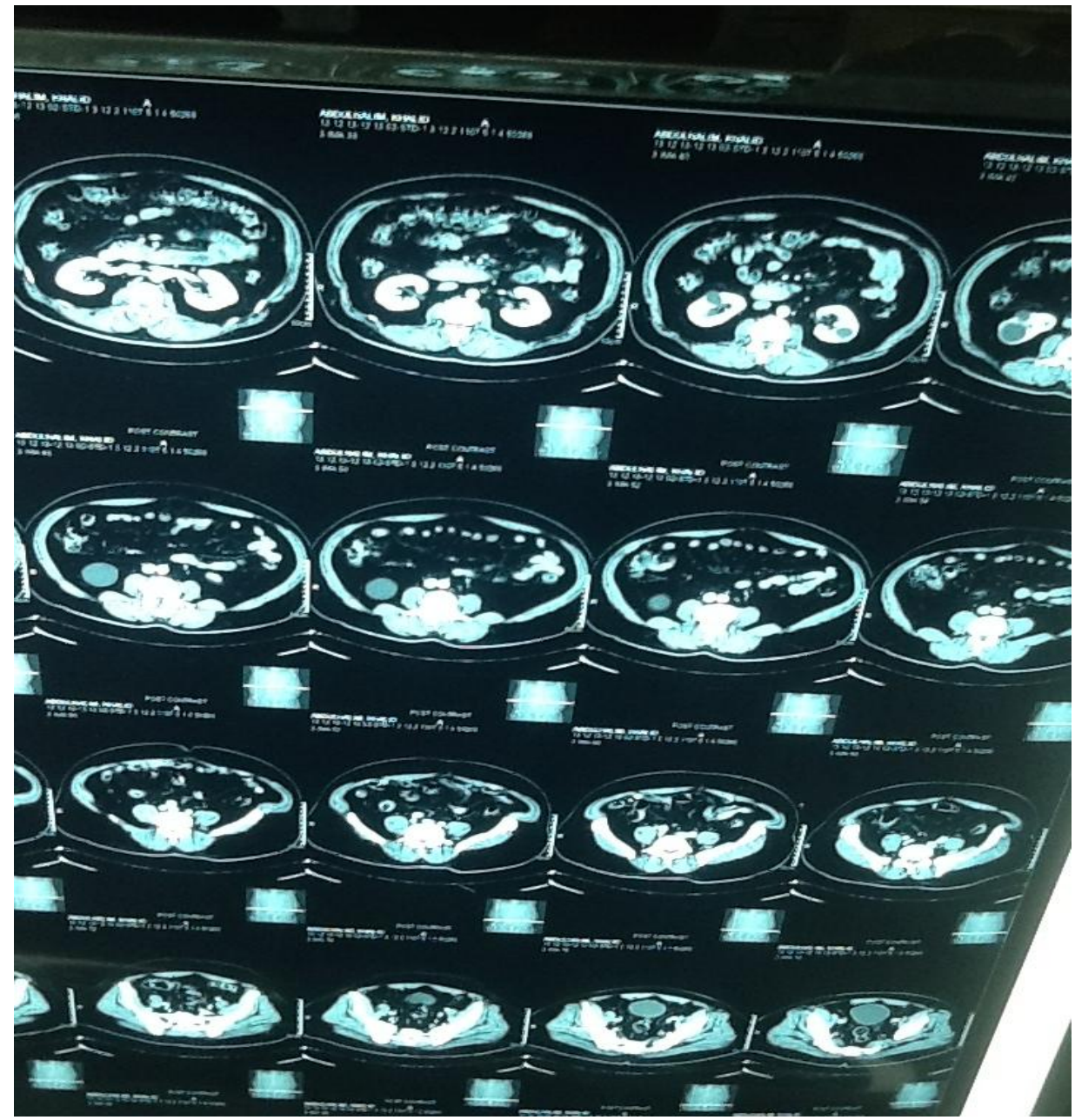

FIGURE 4: CT-scan showing multiple cysts on the kidneys 


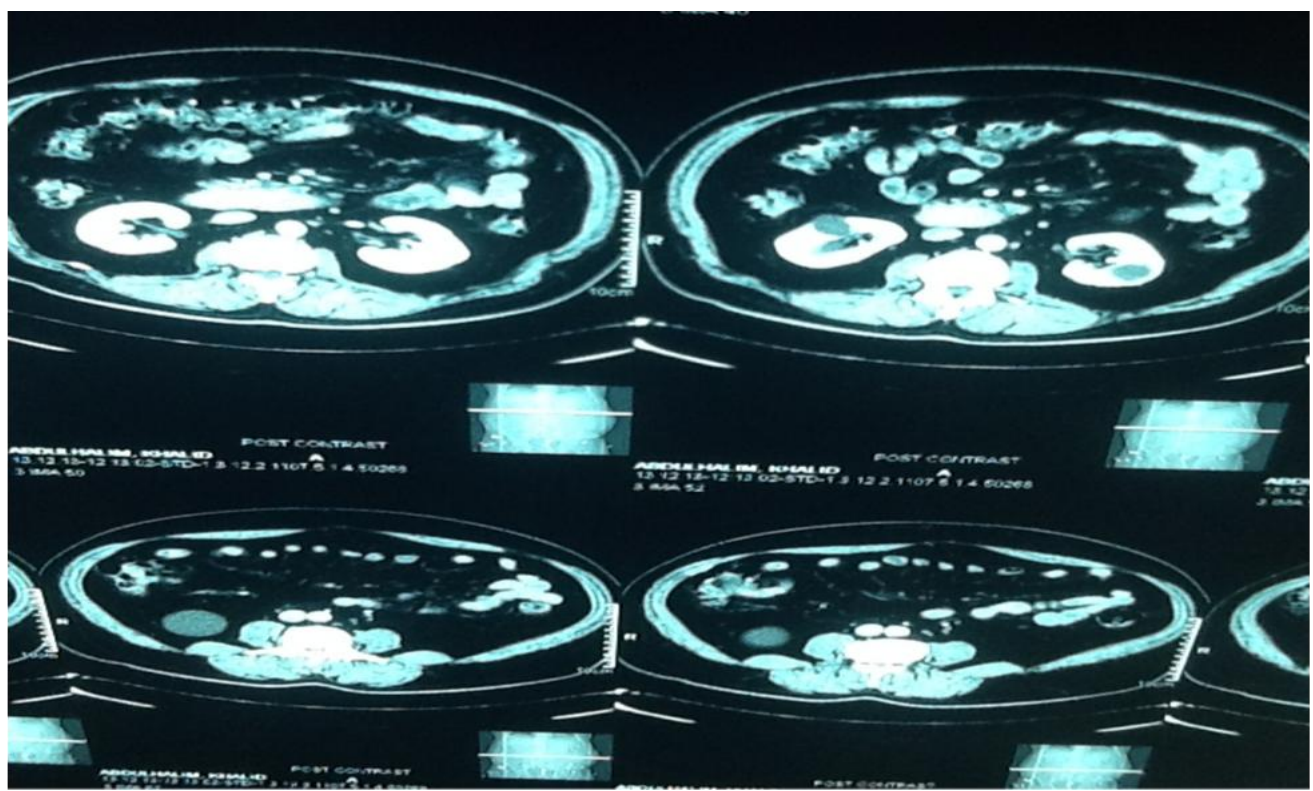

FIGURE 5: CT-scan showing multiple cysts on the kidneys

Lipid profile, liver function test and renal function test were all normal. ECG

The patient was managed by Telmisartan, Amlodipine, Paracetamol and Glucored forte.

This case report illustrates how polycystic kidney disease was diagnosed despite how it presented and being a cause of secondary hypertension.

\section{DISCUSSION:}

Polycystic kidney disease affects 12.5 million people worldwide. It is found in all races and affects men and women equally. ${ }^{3}$

This disease affects people of all ages from neonates to adults, for adults usually it affects on their 50's it is a life threatening condition to some individuals but to some it is asymptomatic, to our patient he is 49 years old symptomatic although his blood pressure is currently controlled but the presence of comorbid diabetes requires him to have several close monitoring.

The average age of end stage renal disease is approximately 55 years ${ }^{4}$, our patient although he is 49 years he has normal renal function test, no any signs of renal impairment apart from presence of renal stones on the right kidney. The risk of developing end stage renal disease include being male, enlarged kidneys, presence of visible blood or detectable protein in urine, or having high blood pressure (particularly before the age of $35 \mathrm{yrs}$ ) ${ }^{4}$, our patient seems to be in high risk of developing end stage renal disease because of the above risk factors, thus requires close monitoring and evaluation and if possible draining of the cysts might be a better option as the part of management for our patient.

It is usually easy to diagnose autosomal dominant polycystic kidney disease (ADPKD) in people who develop flank or abdominal pain and in those who have a family history of ADPKD. An imaging study, such as an ultrasound, is recommended as the first diagnostic test and may reveal multiple cysts on both kidneys. Cysts may also be seen in the liver, pancreas, and spleen. ${ }^{4}$

In people without a family history, ADPKD may be more difficult to diagnose. The diagnosis of polycystic kidney disease may first be suspected based on an imaging test, such as an ultrasound, performed for some other reason. The family history may be negative either because family members developed symptoms at a later age and died of other causes before ADPKD was diagnosed. ${ }^{4}$

Our patient had no family history of ADPKD; the diagnosing was based on presence of flank pain, increase in abdominal size and hypertension, and was confirmed by abdominal pelvic ultrasound and abdominal CT-scan. And the abdominal pelvic ultrasound was done fore mostly to rule out renal stones

Management of polycystic kidney disease involve pharmacological

\section{REFERENCE}

1. Mayo clinic internal medicine

2. Emedicine

3. www.kidney.org/atoz/pdf/polycystic.pdf

4. http://www.uptodate.com/contents/polycystic-kidney-disease-beyond-the-basics 